\title{
Perineural Invasion and TAMs in Pancreatic Ductal Adenocarcinomas: Review of the Original Pathology Reports Using Immunohistochemical Enhancement and Relationships with Clinicopathological Features
}

\author{
Linjuan Zeng ${ }^{1,2^{*}}$, Yubo Guo ${ }^{2 *}$, Jianzhong Liang ${ }^{3 *}$, Shaojie Chen², Peijian Peng1, Qiubo Zhang ${ }^{4}$, Hong Su ${ }^{2}$, \\ Yinting Chen ${ }^{2 \bowtie}$, Kaihong Huang ${ }^{2 \bowtie}$ \\ 1. Department of Oncology, The Fifth Affiliated Hospital of Sun Yat-sen University. The $52^{\text {th }}$ of Meihua East Road, Zhuhai 519000, China \\ 2. Department of Gastroenterology, The Second Affiliated Hospital of Sun Yat-sen University. The 107 th of Yanjiang West Road, GuangZhou 510120, \\ China. \\ 3. State Key Laboratory of Oncology in South China, Sun Yat-sen University Cancer Center. The $651^{\text {th }}$ of Dongfeng Road, GuangZhou 510060, China \\ 4. Department of Gastroenterology, Lihuili Hospital of Ningbo Medical Center, The 57th of Xingning Road, Ningbo 315040, China \\ * These authors contributed equally to this work.
}

$\square$ Corresponding authors: Yinting Chen, Tel: +86 208133 2489, Fax: +86 2081332244 Email: chenyt58@mail.sysu.edu.cn or Kaihong Huang, Tel: +86 208133 2489, Fax: +86 2081332244 Email: huangkh@mail.sysu.edu.cn.

( ) Ivyspring International Publisher. This is an open-access article distributed under the terms of the Creative Commons License (http://creativecommons.org/ licenses/by-nc-nd/3.0/). Reproduction is permitted for personal, noncommercial use, provided that the article is in whole, unmodified, and properly cited.

Received: 2014.07.3I; Accepted: 2014.09.26; Published: 20I4.10.22

\begin{abstract}
Objectives: Tumor-associated macrophages (TAMs) are thought to be involved in the perineural invasion ( $\mathrm{PNI}$ ) process and to be associated with poor prognoses. The associations between TAMs, PNI, and clinicopathological features in pancreatic ductal adenocarcinomas (PDAs) remain to be elucidated. Methods: Fifty-nine PDA patients who had undergone pancreaticoduodenectomy were retrospectively examined. The PNI statuses and TAMs were reviewed following H\&E staining and S-100, CD68, and CDI63 immunohistochemical staining. The relationships between PNI, TAMs, and overall survival and various clinical and histopathologic factors were investigated. Results: PNI was identified in $83 \%$ (49/59) of the cases, the TAM density of the $\mathrm{PNI}^{+}$group was greater than that of the $\mathrm{PNI}^{-}$group, and the infiltrating TAMs around the nerves that were invaded by cancer were much more numerous than those around the nerves without cancer cell invasion. The incidences of PNI, lymph node metastasis, high serum CAI9-9 level, cancers in the body/tail, and advanced pathological stage were associated with shorter OSs. In the $\mathrm{PNI}^{+}$group, lymph node metastasis and high levels of TAM infiltration were associated with worse prognoses. Conclusions: TAMs might enhance PNI, and the incidence of PNI was associated with poor prognosis. $\mathrm{PNI}^{+}$status and high levels of TAM infiltration further worsen the prognosis. Therapies targeting TAMs might represent auxiliary and preventive treatment for PNI in PDA patients.
\end{abstract}

Key words: pancreatic ductal adenocarcinomas, perineural invasion, tumor associated macrophages, immunohistochemical, lymph node metastasis.

\section{Introduction}

Perineural invasion (PNI) is a pathological process in which tumor cells migrate along the nerves, and this process correlates with poor prognoses in certain epithelial cancers including pancreatic ductal adenocarcinoma (PDA) [1]. The reported incidence of PNI in PDAs was varies from $70 \%$ to $100 \%$, but the relationship between PNI and the clinicopathological features of PDAs remain controversial $[2,3]$. It has 
been reported that the incidence of PNI is frequently under-reported due to difficulties in nerve identification, and the use of S-100 immunohistochemical (IHC) staining during pathological diagnosis would help to improve the accuracy of the determination [4-6].

The reasons for PNI in PDAs are not clearly yet but this invasion can be partially explained by the interaction between tumor cells and tumor stromal cells. A variety of nonmalignant stromal cells compose the tumor microenvironment and play pivotal roles in tumor progression, migration and invasion, and PNI. Among these cells, macrophages are the best studied cell type that originates from the circulating monocytes. M1-polarized macrophages are characterized by elevated expression and release of pro-inflammatory factors, such as IL-6 and TNF-a, while M2-polarized macrophages exhibit anti-inflammatory properties and release immunosuppressive cytokines such as IL-10 and TGF- $\beta 1[7,8]$. Tumor-associated macrophages (TAMs) are typically regarded as M2-macrophages in tumor microenvironments, and these cells influence cancer progression and metastasis [9]. Interestingly, the M1 cytokine IL-6 has been confirmed to be a tumor-promoting cytokine, whereas the M2 cytokine IL-10 might be a tumor suppressing factor [10]. Furthermore, researchers have found that TAMs induce epithelial mesenchymal transition (EMT) during the initiation and development of in PDAs and that TAMS not only exhibited M2-phenotypes but also exhibit M1-phenotypes [11].

Evidence from clinical and experimental studies has demonstrated a strong association between TAM density and cancer cell metastasis in several types of cancer, including PDAs [7, 12]. In vitro co-culture and in vivo models have shown that endoneurial macrophages induce the perineural invasion of PDA cells, which suggests that tumor cells, nerve cells, and stromal cells are potential elements in the signaling mechanisms underlying PNI. Autocrine and paracrine mechanisms in tumor cells, tumor stroma, and nerves have also been suggested to be involved in this process $[13,14]$. However, the associations between TAMs, PNI and clinicopathological features remain to be elucidated. Enlightened by the progress explicated above, we performed a retrospective, case-control pilot study to examine the following issues: 1) whether S-100 staining improves the detection of PNI in patients undergoing potentially curative resection of PDAs; 2) whether the TAM density is associated with PNI; and 3) whether the incidence or the severity of PNI is associated with any of the clinicopathological features of PDAs.

\section{Materials and methods}

\section{Tissue sample and data collection}

All of the tissue sample and clinical data collection was approved by the Hospital Research Ethics Committee, and consent was obtained from all patients prior to conducting the research. Fifty-nine of untreated PDAs from September 2004 to December 2011 were studied for the presence or absence of PNI. Additionally, the macrophages within the cancerous lesions and around the nerves were also observed.

The numbers of PDA cases that originated within each subsite of the pancreas were as follows: pancreatic head cancers in 40 patients, and pancreatic body/tail cancers in 19 patients. Patients were excluded if they met any of the following conditions: 1) had received neoadjuvant chemoradiotherapy and/or exhibited surgical margin involvement; and 2) no mention of PNI in the original reports. Thirty-seven of the 59 PDA patients were men, and 22 were women; the patients' ages ranged from 28 to 83 years (mean 59).

The symptoms of abdominal pain and referred lumbar and back pain were reviewed. The degree of preoperative pain was evaluated according to a pain scale (without painkillers). The patients who rated their pain as $0-3$ were defined as grade 1 , the patients who rated their pain as 4-6 were defined as grade 2, and the patients who rated their pain as above 7 were defined as grade 3 . Pathological data, including the cancer stage and grade, lymph node metastasis, and PNI status, were recorded from the original reports via the examination of hematoxylin and eosin (H\&E)-stained sections at the time of surgery. Serum CA19-9 levels, C-reactive protein (CRP) levels, LDH levels and preoperative free glucose $(\mathrm{Glu})$ levels were collected before the operations. All of the patients were followed until death or last contact. For statistical purposes, most of the clinicopathological factors, such as serum CA19-9, LDH, Glu, and CRP levels, were further divided into two groups based on the appropriate normal upper limits. The median TAM value was used to divide the cases into two groups. CD68 low and CD163 low refer to values equal or below the median values for $\mathrm{CD}^{+} 8^{+}$and $\mathrm{CD} 63^{+}$cells, respectively. Similarly CD68high or CD163high refer to values above the medians.

\section{H\&E and immunohistochemistry staining}

Formalin-fixed paraffin-embedded specimens were cut into 10 continuous $6-\mu \mathrm{m}$ sections and stained with H\&E S-100 (DAKO, prediluted), CD68 (DAKO, 1:50 dilution), and CD163 (Abcam, prediluted) IHC according to the manufacturers' protocols. Two independent and blinded pathologists assessed the PNI 
statuses and macrophage counts with a multi-headed microscope.

\section{Evaluation}

Pathological stage was determined according to the seventh edition of the American Joint Committee on Cancer (AJCC). The pathological grades were divided into well, moderately, and poorly differentiated groups (grades 1, 2, and 3). PNI was defined by the identification of tumor cells within any of the three layers of the nerve sheath or tumor foci outside of the nerve involving $\geq 33 \%$ of the nerve's circumference [15]. The degree of PNI was defined microscopically as follows: ne0, no perineural invasion; ne1, perineural invasion that was difficult to identify with only one to three occurrences of lesions; ne2, perineural invasion that was easy to find and between ne1 and ne3; and ne3, perineural invasion that was even easier to find with more massive occurrences of lesions and extension beyond the border of the main tumor mass [14]. We regarded ne1 to ne3 as PNI positive $\left(\mathrm{PNI}^{+}\right)$ and ne0 as PNI negative (PNI-). Both CD68- and CD163-positive staining were indicated by brown membranes/cytoplasm. The macrophages around the nerves were counted over the entire slide under a microscope at a magnification of $400 \times$.

\section{Statistical analyses}

All statistical analyses were performed using the SPSS 17.0 software. The differences between means were examined with two-sample $t$ tests. One-way ANOVAs were used to estimate differences between three or more groups. Survival was calculated using the Kaplan-Meier method. The relationships between PNI and other histopathologic factors were examined using the chi-square test. The probability level of $P<$ 0.05 was considered statistically significant. All reported P-values are two-tailed.

\section{Results}

\section{PNI in the PDAs: Increased incidence upon re-review of the H\&E slides using immuno- histochemical enhancement}

According to the original surgical pathology reports, PNI was found in 32\% (19/59) of the cases. When the original surgical specimen sections were re-stained with H\&E and reviewed by two pathologists, $56 \%(33 / 59)$ of the cases were determined to contain PNI, and $83 \%(49 / 59)$ of the cases were reported as PNI-positive when the S-100stained slides further examined. Of the 49 positive PNI cases, the numbers of PNI foci ranged from 1 to 20 separate foci with a mean of 4.3 foci. Based on the results from the S-100 IHC enhancement and degrees of PNI that were evaluated according to previously reported cri- teria [15], 55\% (27/49) of the cases were ne1, $29 \%$ $(14 / 49)$ of the cases were ne2, and $16 \%(8 / 49)$ of the cases were ne3.

\section{Macrophage infiltration around the nerves in the pathological specimens}

Macrophages are one of the most common inflammatory cell types involved in the host stromal response to cancer cell invasion [16]. To explore the macrophage response to PNI, we evaluated the TAM densities and patterns of macrophage infiltration around the nerves in pathological specimens excised from 59 patients with PDAs. As shown in Figure 1, the macrophages were studied with immunolabeling for the macrophage marker CD68, the M2-type macrophages marker CD163, and S-100 staining of the nerves. Table 1 shows the relationship between the macrophages and PNI. The numbers of $\mathrm{CD}^{+} 8^{+}$and $\mathrm{CD}_{163}{ }^{+}$cells within the PDA lesions in the $\mathrm{PNI}^{+}$ group were higher than those of the PNI- group (105 \pm 3.77 vs. $86 \pm 9.57$ for the CD68 ${ }^{+}$cells, $P=0.04$; and $73 \pm$ 4.83 vs. $46 \pm 8.04$ for the $\mathrm{CD}_{163^{+}}$cells, $P=0.017$, respectively.). Although there was no significant relationship between the macrophage counts and PNI grades, infiltrating $\mathrm{CD}^{+} 8^{+}$and $\mathrm{CD} 63^{+}$cells were much more commonly found around the nerves that had been invaded by cancer than around those that had not $\left(7.92 \pm 0.81\right.$ vs. $4.47 \pm 0.55 \mathrm{CD}^{2} 8^{+}$macrophages/nerve, $P=0.000$, and $4.98 \pm 0.62$ vs. $3.03 \pm 0.57$ $\mathrm{CD}_{163}{ }^{+}$macrophages/nerve, $P=0.001$, respectively). Notably, the nerves in the PDAs lesion that had not been invaded by cancer cells were surrounded by greater numbers of $\mathrm{CD}^{2} 8^{+}$and $\mathrm{CD} 163^{+}$cells than were the nerves in the benign areas of pancreatic tissues (4.47 \pm 0.55 vs. $2.00 \pm 0.71 \mathrm{CD}^{+} 8^{+}$macrophages/nerve, $P=0.000$, and $3.03 \pm 0.57$ vs. $0.23 \pm 0.17 \mathrm{CD} 163^{+}$macrophages/nerve, $P=0.018$, respectively, Figure 2), which indicates that the TAMs enhanced cancer cell invasion along the nerves.

Table I. The relationship between macrophage counts and PNI

\begin{tabular}{lllll}
\hline PNI & $\mathrm{tCD}^{+}$ & $\mathrm{tCD} 163^{+}$ & $\mathrm{p} \mathrm{CD68}{ }^{+}$ & $\mathrm{p} \mathrm{CD163+}$ \\
\hline- & $86(53-136)$ & $46(1-87)$ & $/$ & $/$ \\
+ & $105(47-161)$ & $73(14-142)$ & $/$ & $/$ \\
$P$ value & $0.040^{*}$ & $0.017^{*}$ & $/$ & $/$ \\
$\mathrm{Ne} 1$ & $109(47-161)$ & $78(31-142)$ & $9(2-20)$ & $5(0-12)$ \\
$\mathrm{Ne} 2$ & $96(54-130)$ & $67(14-115)$ & $7(1-20)$ & $5(0-12)$ \\
$\mathrm{Ne} 3$ & $112(80-145)$ & $67(44-97)$ & $7(1-12)$ & $6(1-20)$ \\
$P$ value & 0.281 & 0.499 & 0.771 & 0.434 \\
\hline
\end{tabular}

*Difference is significant at $P<0.05$ level. PNI, peripheral neural invasion. tCD68+, CD68 ${ }^{+}$cells intra-PDAs lesion. tCD163+, CD163+ cells intra-PDAs lesion. pCD68 ${ }^{+}$,

$\mathrm{CD} 68^{+}$cells around nerves. $\mathrm{pCD} 163^{+}, \mathrm{CD} 163^{+}$cells around nerves. 


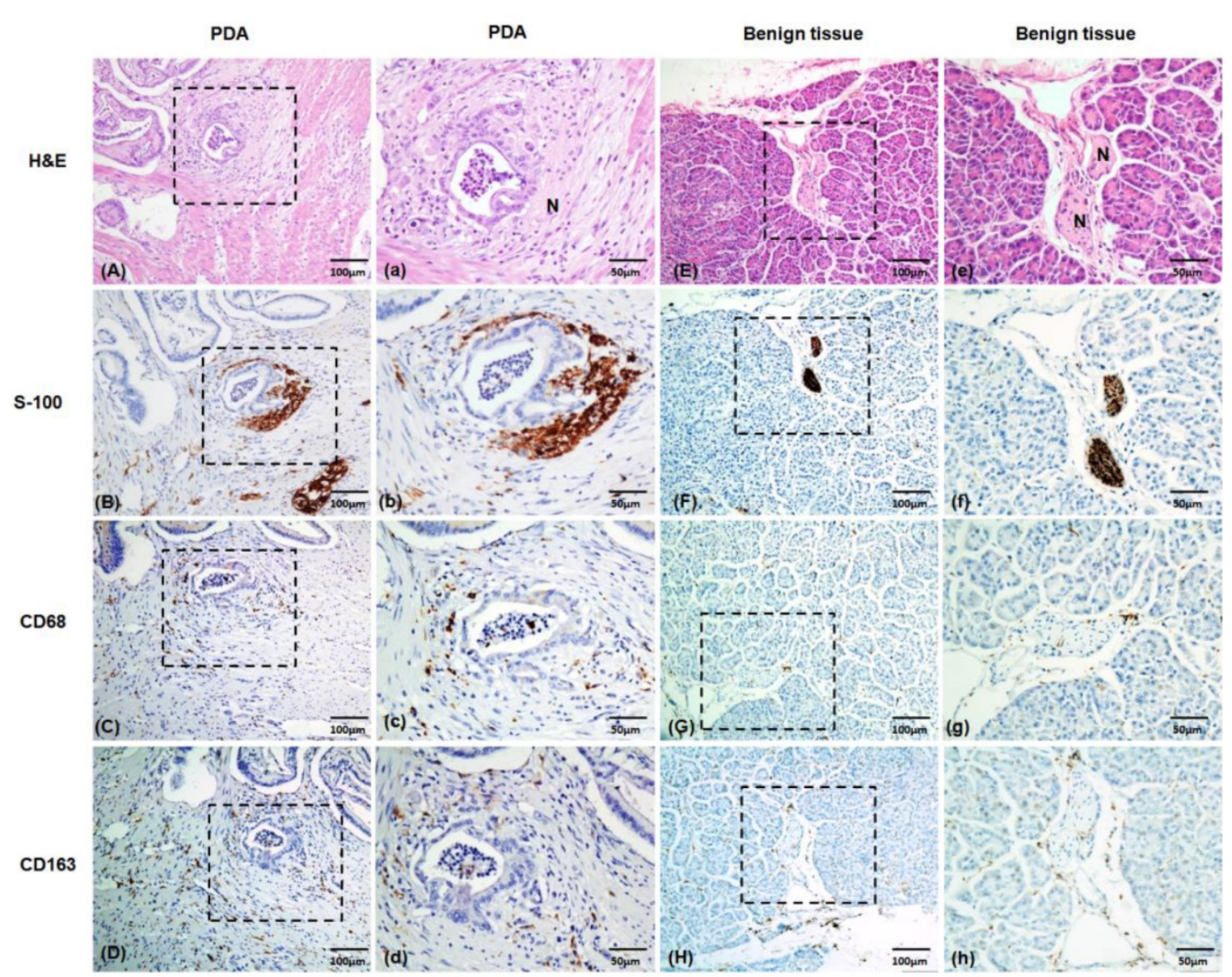

Figure I. Representative images of nerves with $\mathrm{H} \& \mathrm{E}$ and $\mathrm{S}-100$ immunolabeling staining. The macrophages were studied using immunolabeling with the macrophage marker CD68 and the M2-type macrophage marker CDI63. (A), (E) H\&E staining of the nerves. (B), (F) S- 100 immunohistochemical staining of the nerves. (C), (G) CD68 immunohistochemical staining of the macrophages. (D), (H) CDI63 immunohistochemical staining of the macrophages. (A) to $(D)$, Tumor tissue samples with intra-PDA lesions. $(E)$ to $(H)$, Benign pancreatic tissues. (a) to $(h)$ Magnified images of $(A)$ to $(H)$, respectively. PDA, pancreatic ductal adenocarcinoma. $\mathrm{N}$, nerve's bundle. Positive staining is shown in brown.

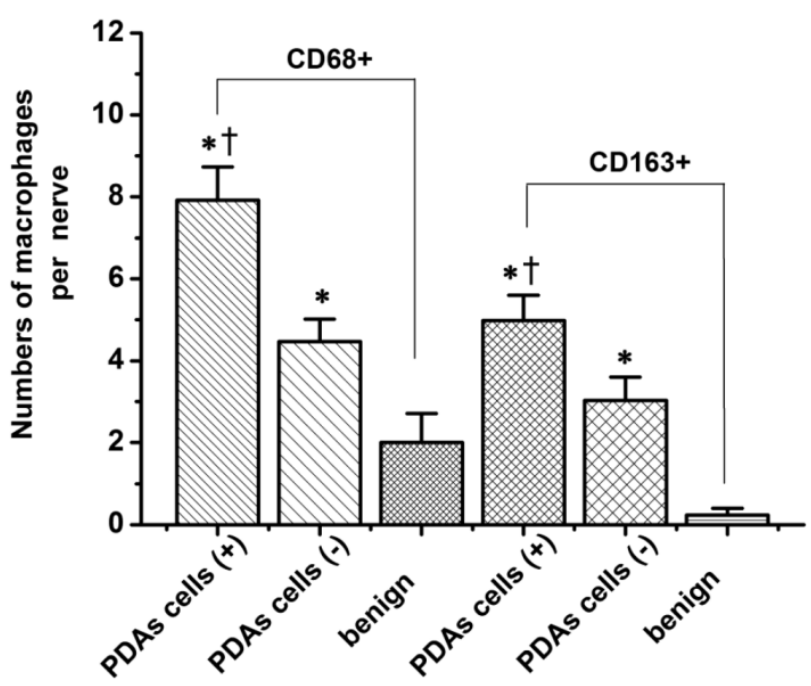

Figure 2. The macrophage counts around nerves with and without cancer cell infiltration. PDA cell (+), nerves infiltrated by PDAs cells; PDA cell (-), nerves not included in a cancer foci without direct contact between them. The data are shown as the means \pm the S.Es $(n=49) . * P<0.05$ compared to PDA cell $(-),+P<0.05$ compared to benign pancreatic tissues. Abbreviations: PDA, pancreatic ductal adenocarcinoma.
There were no significant correlations of PNI with pathological stage, pathological grade, or lymph node metastasis in the PDAs (data not shown). The relationships between various clinicopathological features and PNI in the PDAs are shown in Table 2. The incidence of PNI was significantly correlated with abdominal pain $(P=0.000)$, and the levels of Glu in the patients with PNI were significantly higher than those in the patients without PNI (8.77 \pm 3.80 vs. $6.25 \pm$ 1.03, $P=0.043)$. There were no significant relationships of PNI with serum LDH, CRP, or CA199 levels. The results of univariate analyses of the associations of the prognostic factors with OS are shown in Table 3. The incidences of PNI, lymph node metastasis, high serum CA19-9 level, cancers in the body and tail, and advanced pathological stage were associated with poor prognosis. The mean OS of the PNI- group was significantly longer than that of the $\mathrm{PNI}^{+}$group $(21.20$ \pm 3.36 vs. $13.12 \pm 1.08, P=0.006$ ), but there were no significant differences among the $\mathrm{PNI}^{+}$sub-groups. The mean OSs of the ne1, ne2 and ne3 sub-groups were $14.33( \pm 1.41), 10.64( \pm 1.65)$, and $11.38( \pm 3.14)$, 
respectively $(P>0.05)$. Our results indicate that the incidence but not the severity of PNI affected the prognoses of PDAs. The OS curves (Kaplan-Meier) plotted according to PNI incidence, lymph node metastasis and macrophage counts are shown in Figure 3. Among the patients with PNI, lymph node involvement was associated with worse prognosis $(\mathrm{P}=$ 0.002), and the CD68 ${ }^{\text {high }}$ and CD163 high patients had worse prognoses than did the $\mathrm{CD} 68^{\text {low }}(\mathrm{P}=0.028)$ and CD163 low $(P=0.004)$ patients.

Table 2. The relationship between multiple clinicopathological features and PNI in PDAs.

\begin{tabular}{|l|l|l|l|l|l|l|l|l|l|l|l|}
\hline PNI & \multicolumn{3}{|c|}{$\begin{array}{c}\text { abdominal } \\
\text { pain }\end{array}$} & \multicolumn{2}{c|}{ LDH } & \multicolumn{2}{c|}{ Glu } & \multicolumn{2}{c|}{ CA199 } & \multicolumn{2}{c|}{ CRP } \\
\hline & 1 & 2 & 3 & $\leq 220$ & $>220$ & $\leq 7$ & $>7$ & $\leq 34$ & $>34$ & $\leq 5$ & $>5$ \\
\hline $\mathrm{Ne} 0$ & 6 & 4 & 0 & 9 & 1 & 8 & 2 & 2 & 8 & 5 & 5 \\
\hline $\mathrm{Ne} 1$ & 9 & 17 & 1 & 16 & 11 & 14 & 13 & 8 & 19 & 8 & 19 \\
\hline $\mathrm{Ne} 2$ & 1 & 7 & 6 & 8 & 6 & 6 & 8 & 2 & 12 & 8 & 6 \\
\hline $\mathrm{Ne} 3$ & 0 & 5 & 3 & 5 & 3 & 3 & 5 & 2 & 6 & 3 & 5 \\
\hline $\begin{array}{l}\mathrm{P} \\
\text { value }\end{array}$ & \multicolumn{9}{|c|}{$0.000^{* *}$} & \multicolumn{3}{|c|}{0.341} & \multicolumn{2}{|c|}{$0.043^{*}$} & \multicolumn{2}{|c|}{0.468} & \multicolumn{2}{c|}{0.726} \\
\hline
\end{tabular}

*correlation is significant at $P<0.05$ level

** correlation is significant at $P<0.01$ level

$\mathrm{PNI}$, peripheral neural invasion. Ne, intrapancreatic nerve. LDH, Lactate dehy-

drogenase. Glu, preoperative free glucose. CA19-9, carbohydrate antigen 19-9. CRP, C-reactive protein.

(A)
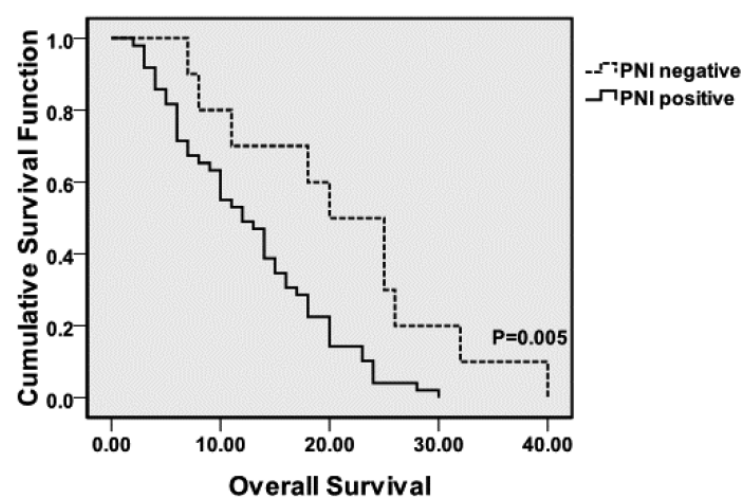

(C)

PNI positive

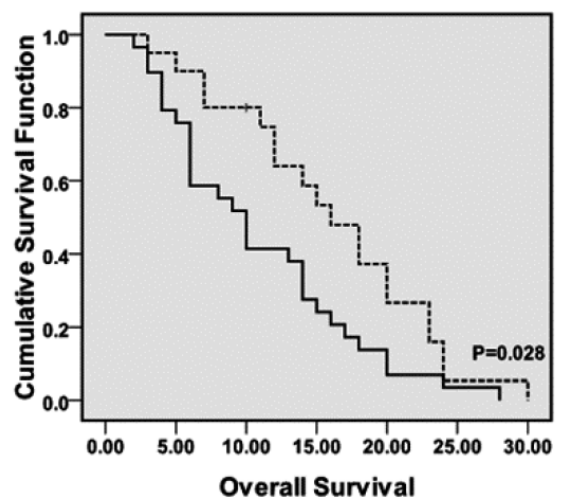

Table 3. Univariate Analysis of Prognostic Factors Associated With OS.

\begin{tabular}{|c|c|c|c|c|}
\hline Factor & Categories & $\mathrm{n}$ & $\begin{array}{c}\text { Median Survival, } \\
\text { mo (mean } \pm \text { SE) }\end{array}$ & $P$ value \\
\hline \multirow{6}{*}{ PNI } & - & 10 & $21.20 \pm 3.36$ & \multirow[b]{2}{*}{$0.006^{* *}$} \\
\hline & + & 49 & $13.12 \pm 1.08$ & \\
\hline & $\mathrm{Ne} 0$ & 10 & $21.20 \pm 3.36$ & \multirow{4}{*}{$0.014^{*}$} \\
\hline & $\mathrm{Ne} 1$ & 27 & $14.33 \pm 1.41$ & \\
\hline & Ne 2 & 14 & $10.64 \pm 1.65$ & \\
\hline & $\mathrm{Ne} 3$ & 8 & $11.38 \pm 3.14$ & \\
\hline \multirow[t]{2}{*}{$\mathrm{LN}$} & - & 26 & $16.81 \pm 1.96$ & \multirow[t]{2}{*}{$0.037^{*}$} \\
\hline & + & 33 & $12.18 \pm 1.14$ & \\
\hline \multirow[t]{2}{*}{ CA19-9 } & $\leq 34$ & 14 & $18.71 \pm 2.36$ & \multirow[t]{2}{*}{$0.034^{*}$} \\
\hline & $>34$ & 45 & $13.18 \pm 1.22$ & \\
\hline \multirow[t]{2}{*}{ CRP } & $\leq 5$ & 24 & $16.33 \pm 2.04$ & \multirow[t]{2}{*}{0.062} \\
\hline & $>5$ & 35 & $12.78 \pm 1.57$ & \\
\hline \multirow[t]{2}{*}{ Tumor site } & head & 40 & $16.05 \pm 1.34$ & \multirow[t]{2}{*}{$0.042^{*}$} \\
\hline & Body/tail & 19 & $11.21 \pm 1.84$ & \\
\hline \multirow[t]{4}{*}{ Stage } & 1 & 3 & $20 \pm 2.78$ & \multirow{4}{*}{$0.002^{*}$} \\
\hline & 2 & 43 & $15.8 \pm 1.28$ & \\
\hline & 3 & 4 & $12.0 \pm 2.04$ & \\
\hline & 4 & 9 & $6.92 \pm 1.68$ & \\
\hline
\end{tabular}

*correlation is significant at $P<0.05$ level.

** correlation is significant at $P<0.01$ level

$\mathrm{SE}$, standard error. OS, overall survival. PNI, peripheral neural invasion. $\mathrm{Ne}$, intrapancreatic nerve. LN, lymph node metastasis. CA19-9, carbohydrate antigen 19-9. CRP, C-reactive protein.
(B) PNI positive

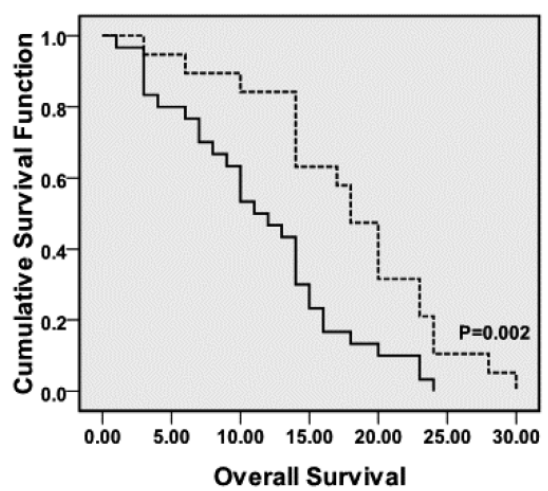

(D)

PNI positive

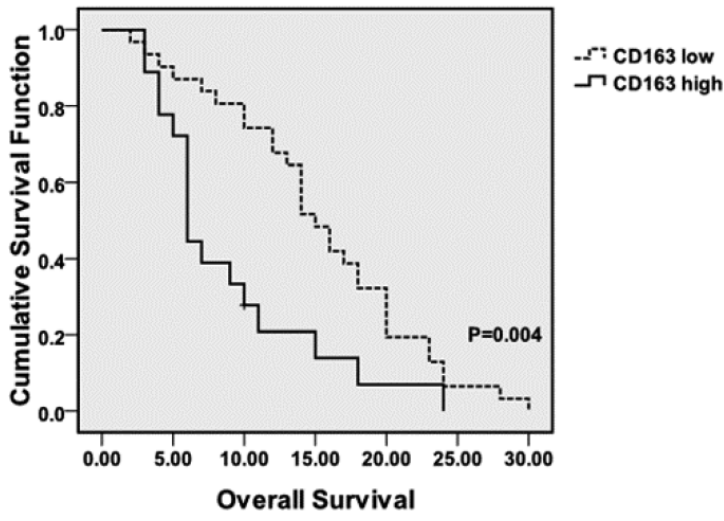

Figure 3. (A) Overall survival curves (OS) for the PDA patients according to the PNI incidence. The OS periods were significantly shorter for the patients with PNI than for the patients without PNI. Among the PNI ${ }^{+}$patients, lymph node metastasis (B), CD68 high $(C)$ and CDI63 high $(D)$ were associated with poorer prognoses. Abbreviations: PNI, peripheral neural invasion. LN, lymph node metastasis. 


\section{Discussion}

Although some reports have shown 100\% PNI in PDAs, others have reported PNI incidences of only $70 \%$, and the values from our original reports were even lower. The accuracy of PNI detection affects further evaluations of PNI and other factors. Therefore, in the present study, we first re-reviewed the slides following routine H\&E staining S-100 immunohistochemical enhancement. Our results showed that the incidences of PNI in the original reports were much lower than those that resulted from our re-review of the slides. This difference might be partially attributable to the differences in the sites of the samples; however, the focused nature of this study is the most likely reason for these differences. In the present study, we concentrated solely on the detection of PNI. Our re-review results, particularly those based on the use of the S-100-stained methodology, reveled a greatly increased incidence of PNI. Because there are no concrete guidelines regarding the reporting of PNI in PDAs, we recommend the review of greater numbers of slides and the use of the S-100 staining methodology when PNI evaluation is necessary for malignancies for which PNI status affects prognosis.

Second, we observed macrophages in the cancer lesions using IHC. In vitro experiments have shown that the addition of stromal cells to the co-culture system results in increased neurite outgrowth and cancer cell colony formation [13]. These findings suggests that tumor cells, nerve cells, and stromal cells might be the different cellular elements that are involved in PNI and that autocrine and paracrine mechanisms potentially underlie PNI. The associations between TAM densities and poor prognoses in several types of cancer, including PDA, have been intensively studied [17-19]. The results of our previous study revealed that the numbers of macrophages in PDA samples are much greater than the numbers in chronic pancreatitis (unpublished data). In the present study, the number of macrophages around the nerves that had been invaded by cancer was significantly greater than that around the nerves that had not been invaded by cancer. In contrast, macrophages were rarely found around the nerves in the benign tissues. Interestingly, even the nerves in the cancer lesions without cancer cell invasion were also surrounded by macrophages, particularly $\mathrm{CD}_{163}{ }^{+}$macrophages. This phenomenon might be explained by a paracrine loop between tumor cells and macrophages. Earlier studies have demonstrated that tumor cells recruited macrophages by secreting colony-stimulating factor- 1 (CSF-1) and the migration and invasion of tumor cells is simultaneously enhanced by macrophage-produced epidermal growth factor (EGF). When tumor cells and macrophages are co-cultured with dorsal root ganglia (DRGs), macrophage recruitment occurs rapidly and is followed by the onset of neural invasion [13].

We next evaluated the associations of PNI with pathological stage, pathological grade, lymph node metastasis, and various other clinicopathological features. PNI was correlated with high Glu levels. Although the mechanism by which hyperglycemia promotes PNI in PDAs remains unclear, it is believed that hyperglycemia promotes cancer cell proliferation, migration and interaction with nerves. In contrast, axons and nerves tend to degenerate or demyelinate in hyperglycemic microenvironments, which renders their resistance to cancer cell invasion insufficient [20, 21].

TAMs are characterized by elevated expressions of various pro- and anti-inflammatory cytokines, such as IL-6, IL-10. Previous studies have shown that IL-6 is essential for breast and pancreatic epithelial cell transformation and for the maintenance of the transformed state potentially due to the activation of the Stat3/Socs3 signal transduction pathway by IL-6 [22-24]. Cytokines, principally IL-6, regulate CRP production, and serum CRP levels have been demonstrated to reflect inner IL-6 activity in several diseases, particularly myeloma [25]. Previous studies have shown that serum CRP level is an independent prognostic marker of various diseases including PDAs [26]. Our results revealed that the mean OS time of the patients in the CRP level $\leq 5$ group was $16.33( \pm$ 2.04) months, while this time was only $12.78( \pm 1.57)$ months in the CRP level $>5$ group. Although this difference was not statistically significant $(P=0.062)$, we believe that the inclusion of a greater number of samples would have produced a significant difference. We hypothesized that TAMs play a role in the regulation of serum CRP concentrations via IL-6 production, but we failed to identify a correlation between these two factors. This lack of association might be because IL-6 was excreted primarily by the M1-macrophages, and TAMs are composed of both M1- and M2-macrophages. Some researchers have reported an inverse correlation between macrophage and CRP level in PDAs, which is indicative of likely complexity of the mechanism responsible for the production of CRP in PDAs [12].

Finally, the cumulative survival function was examined. PNI-positive status was found to independently and negatively affect OS; additionally lymph node metastasis and high grade TAM infiltration into the cancer lesion might worsen the prognoses of PDAs with PNI.

In conclusion, the present study demonstrated that the TAM density of the $\mathrm{PNI}^{+}$group was higher than that of the PNI- group and that infiltrating TAMs 
were much more common around the nerves that had been invaded by cancer than the nerves that had not, which suggests that TAMs enhance PNI. Therapies targeting TAMs might represent auxiliary and preventive treatment for PNI in PDA patients.

\section{Abbreviations}

$\begin{array}{ll}\text { CA19-9: } & \text { Carbohydrate antigen 19-9 } \\ \text { CRP: } & \text { C-reactive protein } \\ \text { CSF-1: } & \text { Colonystimulating factor-1 } \\ \text { DRG: } & \text { Dorsal root ganglion } \\ \text { EMT: } & \text { Epithelial mesenchymal transition } \\ \text { EGF: } & \text { Epidermal growth factor } \\ \text { Glu: } & \text { Glucose } \\ \text { H\&E: } & \text { Hematoxylin and eosin } \\ \text { IHC: } & \text { Immunohistochemical } \\ \text { IL-6: } & \text { Interleukin-6 } \\ \text { LDH: } & \text { Lactate dehydrogenase } \\ \text { OS: } & \text { Overall survival } \\ \text { PDAs: } & \text { Pancreatic ductal adenocarcinomas } \\ \text { PNI: } & \text { Perineural invasion } \\ \text { TAMs: } & \text { Tumor associated macrophages }\end{array}$

\section{Acknowledgments}

This work was supported by the National Natural Science Foundation of China (Grant Nos. $81072045,81302140)$, the Medical Science Foundation of GuangDong Province (Grant No. A2014262), the Scientific-Technologic Foundation of ZhuHai City, China (Grant No. 2013D0401990026), and the Medical Science Foundation of Sun Yat-sen University (Grant No.14ykpy29).

\section{Competing Interests}

There is no financial interest to report that might pose a potential, perceived or real conflict of interest.

\section{References}

1. Hirai I, Kimura W, Ozawa K, et al. Perineural invasion in pancreatic cancer. Pancreas. 2002; 24:15-25.

2. Pawlik TM, Gleisner AL, Cameron JL, et al. Prognostic relevance of lymph node ratio following pancreaticoduodenectomy for pancreatic cancer. Surgery $2007 ; 141: 610-8$.

3. Shimada K, Nara S, Esaki M, et al. Intrapancreatic nerve invasion as a predictor for recurrence after pancreaticoduodenectomy in patients with invasive ductal carcinoma of the pancreas. Pancreas. 2011; 40:464-8.

4. Kurtz KA, Hoffman HT, Zimmerman MB, et al. Perineural and vascular invasion in oral cavity squamous carcinoma: increased incidence on re-review of slides and by using immunohistochemical enhancement. Arch Pathol Lab Med. 2005; 129:354-9.

5. Ceyhan GO, Liebl F, Maak M, et al. The severity of neural invasion is a crucial prognostic factor in rectal cancer independent of neoadjuvant radiochemotherapy. Ann Surg. 2010; 252:797-804.

6. White M, Foulis AK, Smith G, et al. The role of S100 staining in the pathological assessment of perineural invasion in rectal cancer. Colorectal Dis. 2014; 16:71-2.

7. Yoshikawa K, Mitsunaga S, Kinoshita T, et al. Impact of tumor-associated macrophages on invasive ductal carcinoma of the pancreas head. Cancer Sci. 2012; 103:2012-20.

8. Grivennikov SI, Greten FR, Karin M. Immunity, inflammation, and cancer. Cell. 2010; 140:883-99.

9. Sica A, Allavena P, Mantovani A. Cancer related inflammation: the macrophage connection. Cancer Lett. 2008; 267:204-15.
10. Pestka S, Krause CD, Sarkar D, et al. Interleukin-10 and related cytokines and receptors. Annu Rev Immunol. 2004; 22:929-79.

11. Helm O, Held-Feindt J, Grage-Griebenow E, et al. Tumor-associated macrophages exhibit pro- and anti-inflammatory properties by which they impact on pancreatic tumorigenesis. Int J Cancer. 2014; 135:843-61.

12. Jamieson NB, Mohamed M, Oien KA, et al. The relationship between tumor inflammatory cell infiltrate and outcome in patients with pancreatic ductal adenocarcinoma. Ann Surg Oncol. 2012; 19:3581-90.

13. Cavel O, Shomron O, Shabtay A, et al. Endoneurial macrophages induce perineural invasion of pancreatic cancer cells by secretion of GDNF and activation of RET tyrosine kinase receptor. Cancer Res. 2012; 72:5733-43.

14. Koide N, Yamada T, Shibata R, et al. Establishment of perineural invasion models and analysis of gene expression revealed an invariant chain (CD74) as a possible molecule involved in perineural invasion in pancreatic cancer. Clin Cancer Res. 2006; 12:2419-26.

15. Liebig C, Ayala G, Wilks JA, et al. Perineural invasion in cancer: a review of the literature. Cancer. 2009; 115:3379-91.

16. Clark CE, Hingorani SR, Mick R, et al. Dynamics of the immune reaction to pancreatic cancer from inception to invasion. Cancer Res. 2007; 67:9518-27.

17. Condeelis J, Pollard JW. Macrophages: obligate partners for tumor cell migration, invasion, and metastasis. Cell. 2006; 124:263-6.

18. Pollard JW. Tumour-educated macrophages promote tumour progression and metastasis. Nat Rev Cancer. 2004; 4:71-8.

19. Mantovani A, Marchesi F, Porta C, et al. Inflammation and cancer: breast cancer as a prototype. Breast. 2007; 16 Suppl 2:S27-33.

20. Li J, Ma Q. Hyperglycemia promotes the perineural invasion in pancreatic cancer. Med Hypotheses. 2008; 71:386-9.

21. Li J, Ma Q, Liu H, et al. Relationship between neural alteration and perineural invasion in pancreatic cancer patients with hyperglycemia. PLoS One. 2011; 6:e17385.

22. Liu CY, Xu JY, Shi XY, et al. M2-polarized tumor-associated macrophages promoted epithelial-mesenchymal transition in pancreatic cancer cells, partially through TLR4/IL-10 signaling pathway. Lab Invest. 2013; 93:844-54.

23. Bharadwaj U, Marin-Muller C, Li M, et al. Mesothelin overexpression promotes autocrine IL-6/sIL-6R trans-signaling to stimulate pancreatic cancer cell proliferation. Carcinogenesis. 2011; 32:1013-24.

24. Lesina M, Kurkowski MU, Ludes K, et al. Stat3/Socs3 activation by IL-6 transsignaling promotes progression of pancreatic intraepithelial neoplasia and development of pancreatic cancer. Cancer Cell. 2011; 19:456-69.

25. Bommert K, Bargou RC, Stuhmer T. Signalling and survival pathways in multiple myeloma. Eur J Cancer. 2006; 42:1574-80.

26. Haas M, Heinemann V, Kullmann F, et al. Prognostic value of CA 19-9, CEA, CRP, LDH and bilirubin levels in locally advanced and metastatic pancreatic cancer: results from a multicenter, pooled analysis of patients receiving palliative chemotherapy. J Cancer Res Clin Oncol. 2013; 139:681-9. 